\title{
Recent finding and new technologies in nephrolithiasis: a review of the recent literature
}

\author{
Marco Rosa ${ }^{*}$, Paolo Usai ${ }^{2}$, Roberto Miano ${ }^{3}$, Fernando J Kim, Enrico Finazzi Agrò ${ }^{3}$, Pierluigi Bove ${ }^{3}$ and \\ Salvatore Micali ${ }^{4}$ on the behalf of International Translational Research in Uro-Sciences Team (ITRUST)
}

\begin{abstract}
This review summarizes recent literature on advances regarding renal and ureteral calculi, with particular focus in areas of recent advances in the overall field of urolithiasis. Clinical management in everyday practice requires a complete understanding of the issues regarding metabolic evaluation and subgrouping of stone-forming patients, diagnostic procedures, effective treatment regime in acute stone colic, medical expulsive therapy, and active stone removal. In this review we focus on new perspectives in managing nephrolitihiasis and discuss recentadvances, including medical expulsive therapy, new technologies, and refinements of classical therapy such as shock wave lithotripsy, give a fundamental modification of nephrolithiasis management. Overall, this field appears to be the most promising, capable of new developments in ureterorenoscopy and percutaneous approaches. Further improvements are expected from robotic-assisted procedures, such as flexible robotics in ureterorenoscopy.
\end{abstract}

Keywords: Nephrolithiasis, New technologies, Diagnostic procedures, Risk factors, Ureterorenoscopy, Roboticassisted surgery, Shock wave lithotripsy

\section{Review Introduction}

Treating patients with urolithiasis is part of the everyday urological practice. An excellent clinical management involves a complete knowledge of issues regarding metabolic evaluation and subgrouping of stone-forming patients, diagnostic procedures, an effective treatment regime in acute stone colic, medical expulsive therapy, and active stone removal. In the 1980s, results of revolutionary technology such as shock wave lithotripsy (SWL) dramatically changed the therapeutic panorama of lithiasis, while open surgery was disappearing. Today the most invasive procedure for patients with significant stone burden is percutaneous nephrolithotomy (PNL). Furthermore, over the past decade, profound advances in endoscope design, durability, and accessories revolutionized the field of minimally invasive therapy.

Here, we review recent advances in the management of stone disease. We have specifically focused on the following sections:

\footnotetext{
* Correspondence: marcorosa1983@gmail.com

'Department of Urology, University of Modena, Via del Pozzo, 71-41124, Modena, Italy

Full list of author information is available at the end of the article
}

a) epidemiology and risk factors;

b) metabolic evaluation and medical therapy;

c) diagnostic procedures;

d) SWL;

e) surgery, endoscopic procedures, and robot assisted procedures.

\section{Epidemiology and risk factors}

International epidemiological data suggest that the incidence and prevalence of stone disease is increasing [1]. Recent data analysis show a higher prevalence in white population and stronger associations of prevalent kidney stone disease with increased triglycerides, older age, and gallstone disease in African Americans compared to whites, whereas male gender showed stronger association in whites; a dramatic increase of prevalence in female populations is also observed [2]. There was a significant increase in the incidence of kidney stones in children between 1996 and 2007 [3].

Recent papers focused on the most prominent metabolic issues of urolithiasis affecting an ever increasing number of people in developed countries: obesity, diabetes mellitus, hyperuricemia, and metabolic syndrome [4-9]. All these pathologic entities are strongly correlated 
with stone-former patients. After calcium-rich diets were found not to correlate with increased risk of stone formation, whereas calcium and Vitamin D supplementation played a pivotal role in stone-former patients [10]. The protecting role of adequate diet characterized by a high intake of fluids, fruits, and vegetables, a low consumption of salt and protein and a balanced intake of calcium, fats, and carbohydrates constitutes an efficacious approach to the prevention and treatment of this illness $[5,11]$.

\section{Metabolic evaluation and medical therapy}

Identification of metabolic risk factors and correct interpretation of collected data play an important role in managing stone patients and preventing recurrence wherever possible. The new edition of the European Association of Urology (EAU) Guidelines on Urolithiasis includes a useful system of subgrouping stone-forming patients into different categories, based on the type of stone and the severity of symptoms of the disease and also includes a simplified overview of the principles of analytical work-up [12]. Other authors underlined the fundamental role of metabolic work-up in high-risk stone formers [13] and children [14,15]. Still controversial is the role of urology specialists in fields where the nephrologist often plays a major role.

Since patient compliance largely influences medical treatment outcomes, adequate patient information regarding drinking and dietary recommendations plays a major role $[12,13,16]$. Dietary and drinking advice should always be considered before any pharmacological therapy. Correct dietary regimes should never be abandoned even when a pharmacological approach is started.

Various therapeutic tools were used in order to reduce the risk of recurrent calcium stones, that may result in stabilization of stone disease and prevention of the need for further surgical procedures for stone removal [16,17]. Turk et al. gives a brilliant effort to summarize all the suggested treatments and recommendations [12].

\section{Alkaline citrate}

Alkalinizations of tubular cells is the most important factor that results in an increased citrate excretion with only a small fraction of citrate preparations excreted with urine. Citrate calcium chelation reduces ion-activity products of both calcium oxalate and calcium phosphate and inhibits growth and aggregation/agglomeration of these crystals [12]. Thus citrate dilate lithogenesis promotes urinary alcalinization (reducing uric acid supersaturation) and increases cystine solubility. Citrate supplementation plays a fundamental role particularly in patients with hypocitraturia, which constitutes $20 \%$ of all stone formers $[12,18,19]$. Various citrate preparations (sodium potassium citrate, potassium citrate, potassium magnesium citrate, potassium bicarbonate, and sodium bicarbonate) were known to reduce the risk in stoneformer patients. Findings based on randomized studies show that potassium citrate has a greater potential for preventing recurrence than does sodium potassium citrate [20-26]. When oral intake of citrate preparations is unpleasant for the patient, lemon or orange juice could be a valuable option, the latter being a better alkalinising and citraturic agent [21-23]. Citrate supplementation is also useful to considerably decrease stone formation risk that is correlated with prolonged bed rest [20].

\section{Thiazides and thiazide-like agents}

After the initial report by Yendt in 1970 we have more than 30 years of clinical experience with thiazides for calcium stone prevention $[27,28]$. The aim of thiazide treatment is to reduce calcium excretion in hypercalciuric patients (which constitutes around half of stone formers). This effect is thought to be mediated by an increased reabsorption of calcium in the proximal and distal part of the nephron [27-29]. Idiopathic hypercalciuria is a common disorder in children and can present with a range of clinical presentations such as hematuria, voiding dysfunction, flank pain, abdominal pain, nephrolithiasis, urinary tract infection and decreased bone mineral density. Dietary modifications are often sufficient in the management of hypercalciuria. If the symptoms persist or a rare monogenic disorder is present, consideration should be given to medical treatment with a thiazide diuretic and/or citrate therapy [30]. Hydroclorothiazide is usually given at a $25-50 \mathrm{mg}$ dosage once or twice daily. A supplementation with potassium salt (i.e., potassium citrate $3.5-7 \mathrm{mmol}$ twice daily) is needed to counterbalance the thiazide-induced potassium loss and hypocitraturic effect $[12,31,32]$. Thiazide treatment has considerable metabolic side effects: unmasking normocalcaemic hyperparathyroidism, development of diabetes and gout, and erectile dysfunction contribute to a limited patient compliance (50-70\%) and high dropout rate $[12,16,33]$.

\section{Allopurinol}

A xantine-oxidase inhibitor that prevents uric acid production from purine, allopurinol is a commonly used and usually well tolerated anti-gout drug [34]. In urolithiasis patients, treatment is given to counteract the formation of calcium oxalate stones. Allopurinol use in this pathologic condition was introduced following demonstration of a relationship between hyperuricosuria and calcium oxalate stone formation. Allopurinol has been used clinically in patients with or without hyperuricosuria. During the 1980s, Miano et al. [35] performed a placebo-controlled study where treatment with allopurinol was given to hyperuricosuric, calcium oxalate stone formers. Results 
were favorable to the allopurinol group, where $75 \%$ of patients were free of recurrent stones compared with $45 \%$ of the placebo group. Other randomized studies where patients were not selected for hyperuricosuria found no effect on stone formation, thus recent published EAU Guidelines [12] suggest that allopurinol "might be useful for treating patients with hyperuricosuric calcium stone formation" but it "cannot be recommended for patients with other biochemical abnormalities". A new potential pharmacologic therapy for recurrent stone disease is described by Goldfarb et al. Febuxostat, a nonpurine inhibitor of xanthine oxidase (also known as xanthine dehydrogenase or xanthine oxidoreductase) may have advantages over allopurinol and is being tested in a similar protocol, with the eventual goal of determining whether urate-lowering therapy prevents recurrent calcium stones [36]. The major drawback of allopurinol treatment is the occurrence of severe side effects reported with high doses. Adverse effects include Steven-Johnson or Lyell syndrome, vasculitis, hepatitis, and renal failure. Allopurinol should be discontinued immediately in case of cutaneous rush [34].

\section{Phytotherapy}

Various herbal preparations have been used in urolithiasis therapy since ancient times [37]. Grases et al. evaluated the antilithiasic activity of herbal extract and antioxidant flavonoids (catechin and epicatechin) in rats with ethylene glycol induced lithiasis. Herbal preparations and flavonoids showed the ability to prevent papillary and intratubular calcification in the kidney [38]. Phytotherapy was probably clinically efficacious in hastening stone expulsion $(<8 \mathrm{~mm})$ without any observed adverse events [39]. Other herbal preparations show efficacy in stone expulsion after SWL (see further).

\section{Diagnostic procedures and interventional radiology Computed tomography}

Non-contrast computed tomography (NCCT) has been introduced during recent years and has become the well-recognized gold standard and most clinically useful tool for diagnosis of urolithiasis [40-42]. One great advantage is its ability to detect alternative diagnoses and to identify uric acid and xanthine stones that are radiolucent on plain film. This method shows superior [43] specificity and sensitivity compared with Intra Venous Pyelography urography. Combined IVU and CT study allowed correct diagnosis of the underlying cause of delayed excretion or upper urinary tract dilatation in 97\% of cases, reducing time and radiation [44].

Still controversial is its role during follow-up for treated urolithiasis patients and those on observation protocol. Potretzke and Monga [45] suggested that follow-up should be done with plain film radiography if the stone is radio-opaque. Surveillance in children, uric acid stoneformers, and cystine stone-formers should be performed with ultrasonography. Pediatric patients treated for symptomatic urolithiasis could have completed their evaluation and treatment without undergoing NCCT in nearly $90 \%$ of the cases [46].

The usefulness of plain radiography is still under debate. Lamb et al. [47] determined the proportion of stone patients in which management is altered by the interpretation of plain abdominal radiographs (KUB). They observed that on the basis of KUB findings a significant change in patient management occurred. Data shows that KUB offers a significant advantage in treatment planning once the diagnosis has been established by NCCT because of information it provides regarding radio-opacity as well stone size and visibility. Johnston et al. agree if the stone is visible on CT scout film, then the decision to use KUB for follow-up can be made. This minimizes radiation exposure and other costs [48]. But stone features cannot be delivered by NCCT alone. NCCT size estimation of distal ureteral stones versus their actual size was investigated by Kishore and coworkers [49]. Findings show that CT is a poor predictor of the largest stone dimension for distal calculi. Thus caution should be used in patient counseling on the rate of spontaneous passage. Experimental studies using dual energy CT images (ie, scanners that can simultaneously acquire images at different energies) try to offer a routine clinical practice to estimate urinary stone composition based on the density of all constituent voxels [50]. In recent study, the introduction of dualenergy computed tomography systems has significant and unique applications for urologists. Imaging data from these scanners can be used to evaluate composition of urinary calculi [51].

\section{Ultrasound}

Ultrasonography is a well-recognized diagnostic tool and is usually the first imaging modality during diagnostic work-out. Ultrasonography is furthermore a safe and useful option in both the pediatric and pregnant populations, for whom it constitutes the imaging modality of choice. Further studies refined the diagnostic usefulness of ultrasonography in the localization of distal ureter calculi by imaging transrectally and transvaginally [52,53]. Mitterberger et al. placed ultrasound transducers with three-dimensional and volume scanning capabilities, transvaginally in female patients and transrectally in males. The authors were able to exhibit stones with $100 \%$ sensitivity, improving diagnosis in patients examined with transabdominal ultrasound and intravenous urogram (IVU), which together had $81 \%$ sensitivity. 
In vitro study is enveloping a promising modality to facilitate spontaneous clearance of kidney stones and increased clearance of residual stone fragments after surgical management. Shan et al. present a novel method and device to reposition kidney stones using ultrasound radiation force delivered by focused ultrasound and guided by ultrasound imaging. Feasibility of repositioning stones was investigated by implanting artificial and human stones into a kidney-mimicking phantom that simulated a lower pole and collecting system. During experiment, stones were located by ultrasound imaging and repositioned by delivering short bursts of focused ultrasound. Stones were seen to move immediately after delivering focused ultrasound and successfully repositioned from the lower pole to the collecting system [54].

\section{Radiation exposure}

Patients undergoing diagnostic imaging may receive excessive doses of radiation during initial diagnostic and follow-up evaluations. Renal collecting systems can be illustrated more precisely with the advent of multidetector row CT through thinner slices, high speed acquisitions, and enhanced longitudinal spatial resolution resulting in improved reformatted coronal images. On the other hand, a significant increase in exposure to ionizing radiation, especially in the radiosensitive organs, such as the gonads, is a concern with the increased utilization of urinary tract CT [55]. Few studies investigated the effective radiation dose associated with an acute stone episode and short-term follow-up. Ferrandino et al. in a single-institution study found that 205 patients received a dose greater than $20 \mathrm{mSv}$. John et al. found a median radiation dose per stone episode of $5.3 \mathrm{mSv}$, with higher doses in those with renal stones and those who required CT scans and other interventions. Ferrandino suggests that urologists must be cognizant of the radiation exposure to patients and seek alternative imaging strategies to minimize radiation dosages during acute and long-term stone management. [56,57]. In the US, around 60 million CT scans are performed every year [43], raising concern about the amount of radiation delivered. Thus different lower-dose radiation protocols were proposed [56-60]. Results show a high efficacy of lower-dose CT. Unfortunately, studies defined standard and low-dose protocols differently. A standard protocol uses about $180 \mathrm{mAs}$ and low-dose protocol would be performed with about $30 \mathrm{mAs}$. Furthermore, a major role is played by the slice thickness and therefore the patient's time exposure. But low-dose protocols use thicker slices than standard protocols, raising the risk of failure in detecting smaller stones. Memarsadeghi et al. determined that overlapping 3-5 $\mathrm{mm}$ slices could be a sufficient parameter for detection of significant urinary stones [61].
Ciaschini et al. found no significant differences with low dose $(-25 \%$ and $-50 \%)$ examinations for the detection of calculi greater than $3 \mathrm{~mm}$ [62]. Jellison et. al and Jin et al. compared ultra low dose and conventional computerized tomography protocols for detecting distal ureteral calculi [63] and renal calculi [64] in a cadaveric model. Jellison's ultra low dose computerized tomography protocols detected distal ureteral calculi in a fashion similar to that of conventional computerized tomography protocols in a cadaveric model. These protocols may decrease the radiation dose up to $95 \%$. Jin decreased the tube charge from 100 to $30 \mathrm{mAs}$, resulted in similar detection of renal stones respect conventional CT. Dose reduction is also important in pediatric settings. The use of the $80 \mathrm{~mA}$ setting for all children and $40 \mathrm{~mA}$ for children weighing $50 \mathrm{~kg}$ or less does not significantly affect the diagnosis of pediatric renal stones [65].

\section{SWL}

SWL has changed dramatically the management of urolithiasis since the early 1980s. Widespread use of the technology, development of smaller devices, modified indication, and the lower cost of the procedure revolutionized the approach to stone patients. The large amount of sessions performed in the last 25 years allowed for the collection of important data on the indications, contraindications, and adverse effects of the procedure. Krambeck et al. [66] collected data regarding diabetes and hypertension associated with SWL performed with a Dornier HM3 lithotripter. Hypertension incidence was significantly correlated with bilateral procedures, while diabetes was correlated with shock wave number and frequency. The authors suggest that unobserved micro-trauma on the pancreas and kidney could explain the incidence of diabetes and hypertension. On the other side recent study by Chew et al. [67] compare the prevalence of hypertension and diabetes mellitus (DM) in patients treated with an unmodified HM-3 lithotripter (USWL) and a second-generation modified HM-3 lithotripter (MSWL) 20 years ago at their centre in Vancouver with that in the provincial population. No association between lithotripsy and the development of either DM or hypertension in a multivariate analysis. They postulate that the development of renal calculi in our subjects is more indicative of an overall metabolic syndrome where there is increasing evidence that patients with kidney stones get hypertension and diabetes and vice-versa. The development of these diseases is not related to shockwave lithotripsy, but rather to a systemic metabolic dysfunction.

Lee et al. in 2011 propose SWL treatment at a frequency of 60 shocks/min yielded better outcomes, such as a lower number of SWL sessions, and had an increased success rate compared with SWL at 120 shocks/min. On the 
other hand, pretreatment did not impact renal injury. Therefore, SWL treatment at a frequency of 60 shocks/ min could improve treatment efficacy more than that for SWL at 120 shocks/min. [68]. Mazzucchi et al. found no significant differences in the stone-free and complication rates were observed by reducing the total number of impulses from 4000 to 3000 and the frequency from 90 to 60 impulses per minute [69]. Chacko et al. favors a frequency reduction, arguing that $90 \mathrm{sw} /$ min treatment gave better results in terms of stone fragmentation compared to $120 \mathrm{sw} / \mathrm{min}$ [70]. Further reduction of frequency $(30 \mathrm{sw} / \mathrm{min})$ showed a protective effect on renal vessels in an animal model [71]. Furthermore, Tham et al. observed optimal fragmentation by using a short delay time $(20 \mu \mathrm{s})$ between shock waves [72].

In animal models, stepwise power increases (18-20$22 \mathrm{kV}$ ) during treatment gave better results in terms of stone comminution compared with power decreases or leveling ( $96.5 \%$ vs. $89 \%$ vs. $87.6 \%$, respectively). Moreover, Willis et al. proposed a "pre-treatment" of the kidney with low-energy shock waves $(12 \mathrm{kV})$ in order to reduce renal injury [73,74].

Nomograms were introduced by Kanao et al. correlating stone size, location, and numbers to predict stone-free rates after the procedure using a Dornier Lithotripter D [75]. Recently, Nakajiima and Kanao validated the nomograms, finding a remarkable area under the curve (AUC) value of 0.725 [76]. This remarkable effort to predict outcome was until now limited to the Dornier machine. Vakalopoulos [77] avoided this gap by developing a mathematical model to predict extracorporeal shockwave lithotripsy outcomes where predictive equations can be created for different lithotripters. Wiesenthal JD et al. developed a comprehensive nomogram to predict renal and ureteral stone shock wave lithotripsy outcomes, dependent on patient and stone related factors. This factors included stone location, were age, body mass index, stone size, mean stone density $(\mathrm{p}<0.01)$ and skin to stone distance [78].

Shen et al. Perform a systematic review to assess the necessity and complications of DJ stenting before extracorporeal shock wave lithotripsy in the management of upper urinary stones. The systematic review suggested significant advantages of stenting before extracorporeal shock wave lithotripsy compared to in situ extracorporeal shock wave lithotripsy in terms of Steinstrasse. However, stenting did not benefit stone-free rate and auxiliary treatment after extracorporeal shock wave lithotripsy, and it induced more lower urinary tract symptoms [79]. El Assmi found that the presence of hydronephrosis does not affect success rates for distal ureteral stones but increases the number of treatments needed to obtain stone clearance [80].

Recent studies showed the usefulness of CT imaging in predicting fragility and consequently outcomes after SWL.
Such imaging could also offer a considerable amount of information regarding intrarenal anatomy (i.e., lower pole calyx orientation), stone location, and stone composition. Studies by Alon et Garcia Marchinena et al. attempt to characterize stone composition with CT in order to have an indication in the management of calculi suggesting a first-line endoscopic therapy instead of SWL [81,82].

Furthermore, the risk of SWL failure is significantly related to increased radiodensity (signal attenuation) both in vitro and in vivo. Cystine, calcium oxalate monohydrate, and brushite stones are least likely to be fragmented by SWL [83-86].

\section{Post-SWL therapy}

An extensive meta-analysis of medical therapies could be found in the paper by Shuller et al. and Micali and coworkers $[16,87]$. The latter investigated the role of Phyllanthus niruri (a plant belonging to the Euphorbiaceae family used in Brazilian folk medicine by patients with urolithiasis) in SWL and found a positive correlation with lower calyx stone expulsion [88].

The same author [89] and Zheng et al. [90] explore the efficacy of expulsive therapy using nifedipine or tamsulosin, both associated with ketoprofene, after SWL of ureteral stones. They found that nifedipine and ketoprofene association play a significant role in increasing stone free rates for the proximal and middle ureter $(85.7 \%$ vs. $51.7 \%)$ and that tamsulosin and ketoprofen increase stone free rates in distal ureter stones $(82.1 \%$ vs. 57.1\%). Falahatkar S et al. [91] study the role of tamsulosin as adjunctive therapy after extracorporeal shock wave lithotripsy (ESWL) in 150 patients with 4$20 \mathrm{~mm}$ in diameter renal and ureteral stones. Th patients was shared 71 in control group and 70 in case group, treated with Tamsulosin: thei found a statistically significant difference in time of stone passage from onset of treatment (between 20th and 30th day in control group and between 10th and 20th day (50\%) in case group after ESWL). Sighinolfi et al. [92] found that treatment with Tamsulosin after ESWL increases fragments expulsion rate of renal calculi also.

As seen above, citrate supplementation could play an important role in expulsive therapy after SWL. In a study conducted on 96 hypocitraturic children who underwent SWL potassium citrate showed a significant role in decreasing recurrence (7.6\% in citrate arm vs. $34.6 \%$ in placebo) agglomeration of residual fragments [25].

\section{SWL vs. URS}

Debate over the most favorable method still continues and probably will continue for decades. Kijvikai et al. [93] try to offer some balanced consideration on the better treatment for distal ureteral stones. SWL and URS (ureteroscopic lithotripsy) are both considered to 
produce excellent stone free rates (86-90\%), but stones $>10 \mathrm{~mm}$ have better outcomes with endoscopy (73\% vs. 67\%). Aboumarzouk OM et al. [94] agree that, compared with ESWL, ureteroscopic removal of ureteral stones achieves a greater stone-free state, but with a higher complication rate and longer hospital stay. Furthermore, URS plays a unique role during pregnancy or in patients with uncontrolled blood coagulation [93,95].

In conclusion, SWL revolutionized urolithiasis therapy and is often the treatment of choice for many ureteral and renal stones. Moreover, SWL is related to low complication rates. But a balanced choice should always include patient consideration [96].

\section{Digital endoscopes}

At the beginning of this century, ureteroscopy received a new impulse thanks to novel technological refinements such as miniaturization of scope profile, improved maneuverability, and optimized accessory instrumentation. Currently available flexible ureteroscopes have an average tip diameter of about 6.9-7.5 Fr and a mid-shaft diameter of 7.5-9.0 Fr and can be inserted in an intramural ureter without active dilation in most cases [97].

Image quality was also improved by incorporating an optical chip such as a CMOS (complimentary metal oxide semiconductor) or CCD (charge-couple device) at the tip of the ureteroscope together with distal LED light and image processing capabilities. Digital ureteroscopes eliminate the honeycomb effect, and deflection is comparable to traditional fiber-optic endoscopes. In September 2006, Gyrus-ACMI (Southboro, Massachusetts, USA) was the first to introduce a ureteroscope incorporating this technology: the DUR-D ureteroscope (Figure 1). Preliminary reports indicate that the new-generation flexible ureterorenoscopes are more durable than previous ones $[98,99]$. All these enthusiastic reports should be counterbalanced by an awareness of some disadvantages of the new-generation endoscopes. Rigid and flexible digital ureteroscopes are larger in diameter compared to their analog counterparts, and digital technology has higher costs. Thus more research is necessary to evaluate the true advantage of digital technology for ureteroscopy $[100,101]$. Undoubtedly, images produced by digital endoscopes such as DUR-D are of outstanding quality.

Deflection capability is also an important issue. The Storz FlexX2 Wolf Viper allows a $270^{\circ}$ deflection in both directions, while the Olympus P5 allows $270^{\circ}$ in one direction and $180^{\circ}$ in the other. The DUR 8-elite ureteroscope (ACMI) was the first to offer dual primary and secondary active tip deflection that totals $270^{\circ}$. Preliminary reports suggest that secondary deflection is necessary in approximately 20-29\% of cases [102-104], particularly with regard to lower pole access. Although expensive, the holmium: YAG laser is actually the best

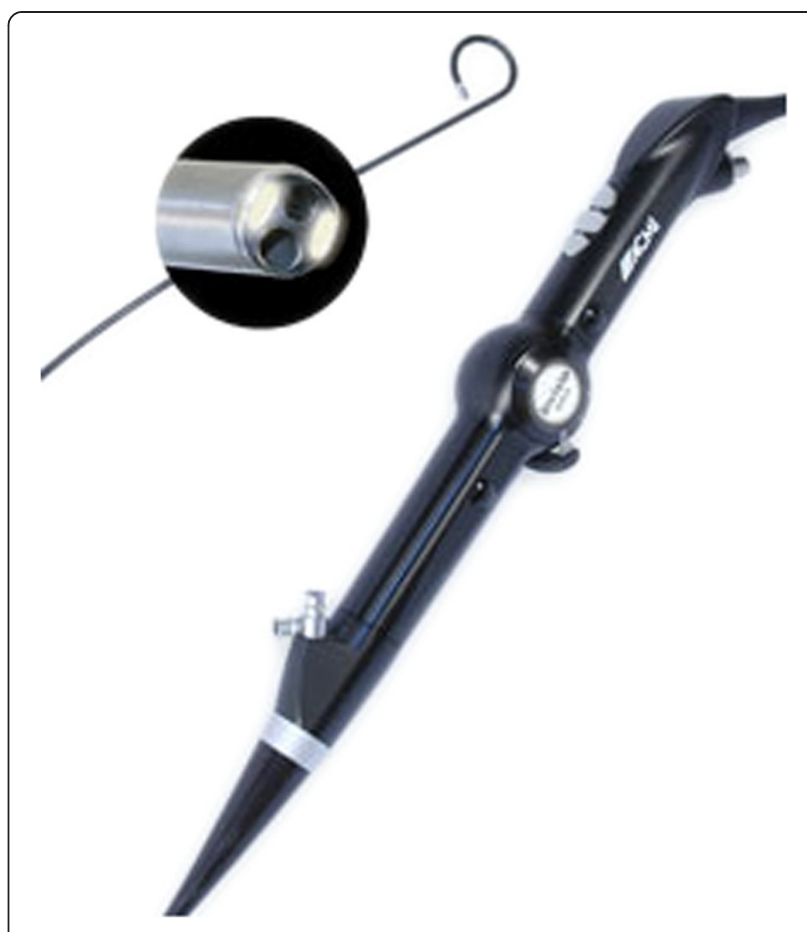

Figure 1 The DUR-D (Olympus) digital and flexible ureteroscope.

intracorporeal lithotripter for the ureter and the benchmark for other energy sources [105-107].

\section{Accessories device}

The ideal basket should be flexible, durable, atraumatic, easily deployed/disengaged/disassembled, and of minimal impact on fluid inflow and tip deflection [108]. Thus, the ideal basket simply does not exist until now. Despite marketing efforts to introduce the "perfect" basket, comparison of four popular basket designs suggested that the more complex wire configurations and deflection capabilities offered no advantage over the simple Cook N-Circle nitinol basket [108,109] (Figures 2 and 3 ).

\section{PNL}

International epidemiological data suggest that the incidence and prevalence of stone disease is increasing and the number of diagnoses and procedures relating to kidney stone disease has increased significantly in the last 10 years in the UK [1]. Since the introduction of SWL during the $1980 \mathrm{~s}$ and development of endoscopic techniques, open surgery is rapidly disappearing. Nowadays PNL represents the most invasive procedure for urolithiasis in significant stone burden patients. In the US and UK, PNL experienced a rapid increase while open surgery is showing a dramatic decreas $[1,110]$.

Percutaneous access is the method of choice in staghorn and complex renal stones with diameters $>2 \mathrm{~cm}$ 


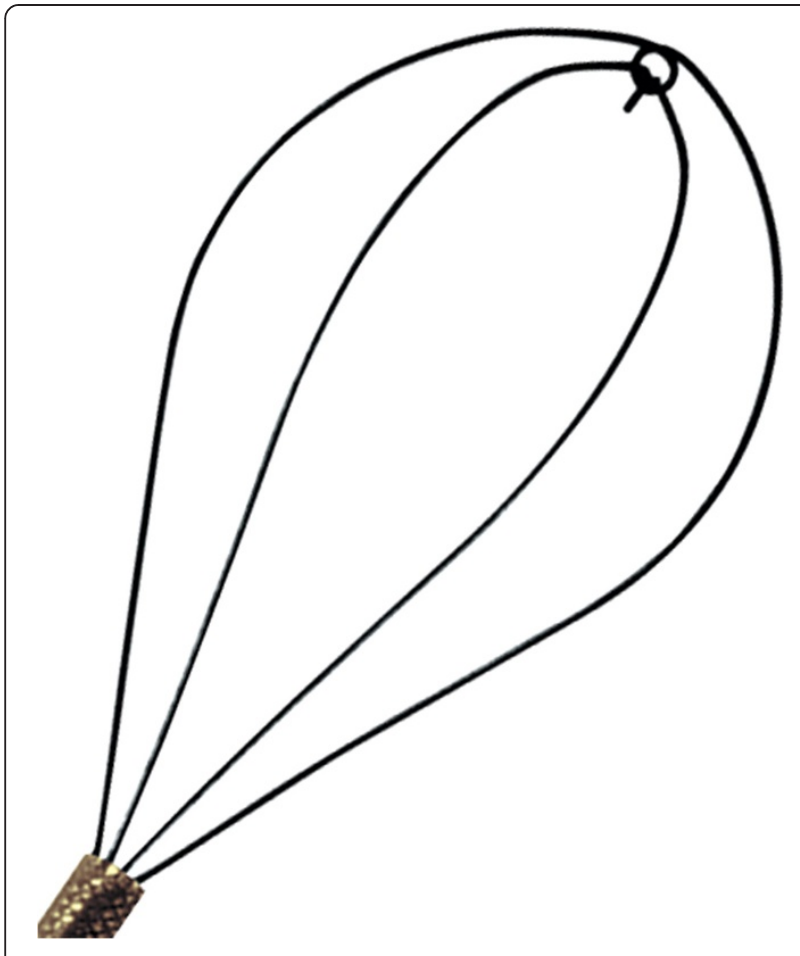

Figure 2 Cook N-Circle nitinol basket.

and for lower pole calculi with diameters $>1 \mathrm{~cm}$ [111]. Debates over patient position (prone or supine) and SWL efficacy versus PNL take place in many journals. PNL is indicated as the first-choice treatment in staghorn and complex renal stones $>2 \mathrm{~cm}$ and for lower calyx stones $>1 \mathrm{~cm}[112]$.

\section{Patient position}

Recent studies proposed and popularized the Valdivia Uria supine position for PNL. Valdivia Uria's original paper dated to 1987 and more than 557 have been performed since then. According to the author, the advantages of the position are a direct and easily access

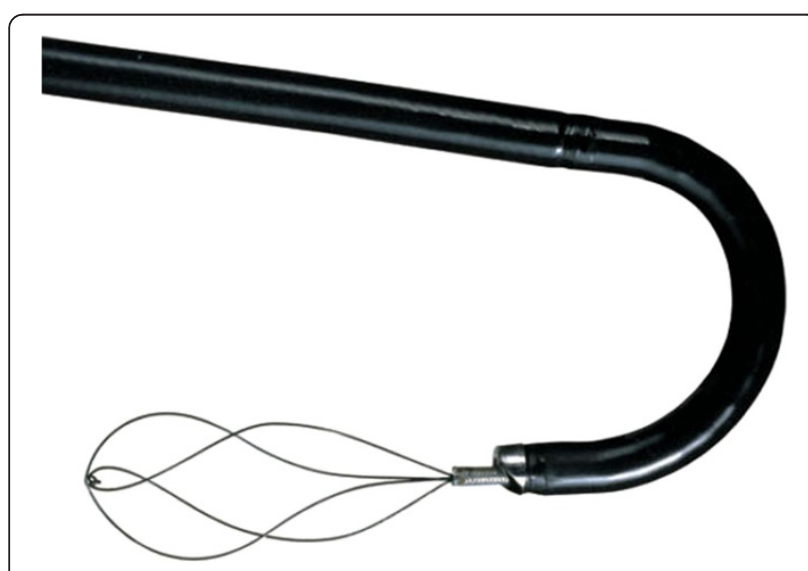

Figure 3 Cook N-Circle nitinol basket on flexible ureteroscope. to anterior oriented calyces, easier access to the bladder, and better stone free rates in comparison to the prone position [113]. A recent review summarized the arguments for and against prone and supine percutaneous nephrolithotomy: the prone position is associated with a decrease in the cardiac index and an increase in pulmonary functional residual capacity. An increased risk of liver and spleen injury exists for upper pole puncture with the patient supine. Potential injury to the colon is greatest during prone lower pole access. A greater surface area for percutaneous access exists with the patient prone. The supine position decreases surgeon radiation exposure and promotes spontaneous stone drainage during the procedure. Two comparative series show that the supine position is associated with significantly shorter operative time. In contrast, noncomparative case series suggest decreased operative time and blood loss when treating staghorn calculi with the patient prone [114].

The supine position is also indicated in a high American Society of Anesthesiologists (ASA) score [115]. Cracco et al. and Kawahara et al. propose ECIRS (Endoscopic Combined IntraRenal Surgery) is a new way of affording PNL in a modified supine position, approaching anteroretrogradely to the renal cavities, and exploiting the full array of endourologic equipment. Supine PNL and ECIRS are not superior to prone PNL in terms of urological results, but guarantee undeniable anesthesiological and management advantages for both patient and operators. In particular, ECIRS requires from the surgeon a permanent mental attitude to synergy, standardized surgical steps, versatility and adherence to the ongoing clinical requirements. ECIRS can be performed also in particular cases, irrespective to age or body habitus. The use of flexible endoscopes during ECIRS contributes to minimizing radiation exposure, hemorrhagic risk and post-PNL renal damage [116,117].

\section{Percutaneous access}

The debate over access numbers still continue. The authors suggest that higher stone free rates could be easily obtained with the use of flexible nephroscopes during the PNL $[118,119]$. Wong et al. reported a stone free rate of $95 \%$ in patients treated with a single percutaneous tract with flexible nephroscopy. The use of a single tract with high stone free rates seems the best achievement in terms of minimal invasiveness and the benchmark for procedure comparison [120]. Akman et al. consider the impact of PCNL using either single or multiple access tracts on renal function, finding similar results. By the way PCNL with multiple accesses is a highly successful alternative with considerable complication rates in the management of staghorn calculi [121]. Li NL et al. propose percutaneous nephrolithotomy 
through the upper pole calix. The access allows greater stone clearance rate due to its easy access into the intrarenal collecting system and can be an ideal approach for PCNL for complicated renal calculi [122].

\section{PNL under local anesthetics}

PNL under local anesthesia is a very attractive method in order to minimize procedure morbidity. Indication should be very strict and must exclude staghorn calculi patients, previous renal surgery, and stone burden $>3.5 \mathrm{~cm}$. Local anesthesia should also include percutaneous tract and renal parenchyma to achieve postoperative pain control $[123,124]$.

\section{Robotic surgery}

Robot-assisted laparoscopic surgery with the daVinci system is immensely popular among urologists. However some of the earliest experiences with robotics in urology were developed by italian group of Bove in the last ' 90 . They performed different kind of procedures (spermatic vein ligation, retroperitoneal renal biopsy, simple nephrectomy and pyeloplasty) with the help of two robots: AESOP for the orientation of the laparoscope and PAKY to perform the percutaneous access [125]. Robotassisted surgery is now well-established in Urology and although not currently regarded as a 'gold standard' approach for any urological procedure, it is being increasingly used for index operations of the prostate, kidney and bladder [126]. Fine movement control and drift-free maintenance of the endoscope distal tip is typically insufficient using manual control to perform complex procedures. The aim of robotic surgery is to allow safer and more homogeneous outcomes with less variability in surgeon performance and reduced occupational radiation exposure. Desai et al. tested on animals a new flexible robotic system for performing retrograde intrarenal surgery [127] and his initial clinical experience on 18 patient was encouraging: all procedures were technically successful without conversion to manual ureteroscopy and the complete stone clearance rate at 2 and 3 months was $56 \%$ and $89 \%$, respectively. At 3 months all patients had stable renal function and unobstructed drainage [128]. According to the authors, potential advantages of the technique include increased range of motion, instrument stability, and improved ergonomics.

The nascent field of flexible robotics appears to be promising. Refinements in software and hardware are needed to allow these systems to be used for natural orifice transluminal surgery. A significant advance in robotic surgery came from URobotics Laboratory at Johns Hopkins (Baltimore, MD, USA), which recently developed the AcuBot robot. This device is a fully actuated driver for needle insertion, spinning, release, and force measurement. This provides an additional needle support guide in close proximity to the skin entry point. The device is the first promising step to a future clinical application of robotic guided percutaneous renal access [129].

\section{"News from the past"}

Recent research by Mariani assesses the feasibility of ureteroscopic monotherapy of renal calculi $>2 \mathrm{~cm}$. Lithotripsy was performed in 75 patients with a single deflection flexible ureteroscope and predominantly electrohydraulic lithotripsy; laser drilling was employed to weaken very hard stones. Stone free status was achieved in $96 \%$ of patients [130]. Recently, similar results was obtained by Hyams et al:: one hundred and twenty patients underwent URS/holmium laser lithotripsy for renal stones of 2 to $3 \mathrm{~cm}$. One hundred and one $(84 \%)$ patients underwent single-stage procedures.

\section{Conclusion}

Urolithiasis is a growing problem in industrialized countries and is often correlated to typical Western pathologies and habits such as diabetes, hypertension, high purine intakes, obesity, and metabolic syndrome. Beside drug treatment, in recent years medical therapies incorporating herbal components known for centuries have been investigated. Data show that it offers new advantage in stone clearance after SWL or in spontaneous stone expulsion. NCCT is nowadays the most useful clinical tool in stone patients. Among all the minimally invasive stone treatments, SWL is always the less invasive one, and stone free rates with SWL are lower than with more invasive treatments. Therapy choice should include these considerations. Development of new technologies offers further advances in well-standardized procedures such as PNL and SWL. Robotics seems the most promising field capable of new developments in ureterorenoscopy and percutaneous approaches.

\section{Competing interests}

The authors declare that they have no competing interests.

Authors' contributions

All authors read and approved the final manuscript.

\section{Author details}

'Department of Urology, University of Modena, Via del Pozzo, 71-41124, Modena, Italy. ${ }^{2}$ Department of Urology, University of Cagliari, Via Aurelio Nicolodi, 1 09123, Cagliari, Italy. ${ }^{3}$ Department of Urology, University "Tor Vergata", Rome, Italy. " Department of Urology, Denver Health Care Center, 777 Bannock Street, Denver, CO 80204-4597, USA.

Received: 8 November 2012 Accepted: 6 February 2013 Published: 16 February 2013

\section{References}

1. Turney BW: Trends in urological stone disease. BJU Int 2011, 24(6):382-386

2. Akoudad S: Correlates of kidney stone disease differ by race in a multiethnic middle-aged population: the ARIC study. Prev Med 2010, 51(5):416-420. 
3. Sas DJ: Increasing incidence of kidney stones in children evaluated in the emergency department. J Pediatr 2010, 157(1):132-137.

4. Chen HS: Increased risk of urinary tract calculi among patients with diabetes mellitus-a population-based cohort study. Urology 2011, 42(8):432-435.

5. Frassetto L: Treatment and prevention of kidney stones: an update. Am Fam Physician 2011, 84(11):1234-1242.

6. Del Valle EE: Metabolic diagnosis in stone formers in relation to body mass index. Urol Res 2011, 38(7):123-127.

7. Maalouf NM: Metabolic syndrome and the genesis of uric acid stones. J Ren Nutr 2011, 21(1):128-131.

8. Hurtes X: Hyperuricemia and uro-nephrological disorders. Presse Med 2011, 40(9 Pt 1):865-868.

9. Jeong IG: Association between metabolic syndrome and the presence of kidney stones in a screened population. Am J Kidney Dis 2011, 58(3):383-388.

10. Wallace RB: Urinary tract stone occurrence in the Women's health initiative (WHI) randomized clinical trial of calcium and vitamin D supplements. Am J Clin Nutr 2011, 94(1):270-277.

11. Meschi T: Lifestyle recommendations to reduce the risk of kidney stones. Urol Clin North Am 2011, 38(3):313-320.

12. Turk C: EAU guidelines on urolithiasis 2011 editon; 2011.

13. Park S: Medical management of urinary stone disease. Expert Opin Pharmacother 2007, 8(8):1117-1125.

14. Dogan HS: Management of pediatric stone disease. Curr Urol Rep 2007, 8(2):163-173.

15. Sarica K: Effect of potassium citrate therapy on stone recurrence and regrowth after extracorporeal shockwave lithotripsy in children. $J$ Endourol 2006, 20(11):875-879.

16. Micali S: Medical therapy of urolithiasis. J Endourol 2006, 20(11):841-847. Rewiew.

17. Zilberman DE: Long-term results of percutaneous nephrolithotomy: does prophylactic medical stone management make a difference? J Endourol 2009, 23(10):1773-1776.

18. Kurtz MP: Dietary therapy for patients with hypocitraturic nephrolithiasis. Nat Rev Urol 2011, 8(3):146-152.

19. Rodgers A: Evening primrose oil supplementation increases citraturia and decreases other urinary risk factors for calcium oxalate urolithiasis. J Urol 2009, 182(6):2957-2963.

20. Zerwekh JE, Odvina CV, Wuermser LA, Pak CY: Reduction of renal stone risk by potassium-magnesium citrate during 5 weeks of bed rest. J Urol 2007, 177(6):2179-2184.

21. Odvina CV: Comparative value of orange juice versus lemonade in reducing stone forming risk. Clin J Am Soc Nephrol 2006, 1(6):1269-1274.

22. Kang DE, Sur RL, Haleblian GE, Fitzsimons NJ, Borawski KM, Preminger GM: Long-term lemonade based dietary manipulation in patients with hypocitraturic nephrolithiasis. J Urol 2007, 177(4):1358-1362.

23. Zuckerman JM: Hypocitraturia: pathophysiology and medical management. Rev Urol 2009, 11(3):134-144.

24. Caudarella R: Urinary citrate and renal stone disease: the preventive role of alkali citrate treatment. Arch Ital Urol Androl 2009, 81(3):182-187.

25. Lojanapiwat: Alkaline citrate reduces stone recurrence and regrowth after shockwave lithotripsy and percutaneous nephrolithotomy. Int Braz J Urol 2011, 37(5):611-616

26. Singh SKBJU: Medical therapy for calculus disease. Int 2011, 107(3):356-368.

27. Yendt ER: Renal calculi. CMAJ 1970, 102(5):479-489

28. Yendt ER: Commentary: renal calculi twenty years later. J Lithotripsy Stone Dis 1990, 2:164-172.

29. Grieff M: Diuretics and disorders of calcium homeostasis. Semin Nephrol 2011, 31(6):535-541.

30. Srivastava T: Diagnosis and management of hypercalciuria in children. Curr Opin Pediatr 2009, 21(2):214-219.

31. Vigen R: Thiazides diuretics in the treatment of nephrolithiasis: are we using them in an evidence-based fashion? Int Urol Nephrol 2011, 43(3):813-819. Epub 2010 Aug 25

32. Reilly RF: The evidence-based use of thiazide diuretics in hypertension and nephrolithiasis. Clin J Am Soc Nephrol 2010, 5(10):1893-1903. Epub 2010 Aug 26

33. Huen SC, Goldfarb DS: Adverse metabolic side effects of thiazides: implications for patients with calcium nephrolithiasis. J Urol 2007 177(4):1238-1243.
34. British National Formulary. UK: BMJ Publication Group London; 2004.

35. Miano L, Petta S, Galatioto GP, Gallucci M: A placebo controlled double-blind study of allopurinol in severe recurrent idiopathic renal lithiasis, Urolithiasis and related clinical research New York. New York: Plenum Press; 1985:521-524.

36. Goldfarb DS: Potential pharmacologic treatments for cystinuria and for calcium stones associated with hyperuricosuria. Clin J Am Soc Nephrol 2011, 6(8):2093-2097. Epub 2011 Jul 14.

37. Tkachuk VN: Phytotherapy in the treatment of ureteral calculi. Urologiia 2009, 12(3):13-15.

38. Grases F, Prieto RM, et al: Phytotherapy and renal stones: the role of antioxidants. A pilot study in wistar rats. Urol Res 2008, in press.

39. Singh I: Prospective randomized clinical trial comparing phytotherapy with potassium citrate in management of minimal burden $(\leq 8 \mathrm{~mm})$ nephrolithiasis. Urol Ann 2011, 3(2):75-81.

40. Dhar M: Imaging in diagnosis, treatment, and follow-up of stone patients. Adv Chronic Kidney Dis 2009, 16(1):39-47.

41. Carter MR: Renal calculi: emergency department diagnosis and treatment. Emerg Med Pract 2011, 13(7):1-17.

42. Mandeville JA: Imaging evaluation in the patient with renal stone disease. Semin Nephrol 2011, 31(3):254-258.

43. Shine S: Urinary calculus: IVU vs CT renal stone? A critically appraised approach. Abdom Imaging 2008, 33(9):41-43.

44. Sebastià C: Usefulness of computed tomography performed immediately after excretory urography in patients with delayed opacification or dilated upper urinary tract of unknown cause. Abdom Imaging 2011, 48(5):81-87

45. Potretzke AM, Monga M: Imaging modalities for urolithiasis: impact on management. Curr Opin Urol 2008, 18:199-204.

46. Wallis MC: Are stone protocol computed tomography scans mandatory for children with suspected urinary calculi? Int Braz J Urol 2011, 37(5):681-682. Division of Pediatric Urology, University of Utah, Salt Lake City, Utah, USA.

47. Lamb AD, Wines MD, Mousa S, Tolley DA: Plain radiography still is required in the planning of treatment for urolithiasis. J Endourol 2008, 22(10):2201-2205.

48. Johnston R: Comparison of kidney-ureter-bladder abdominal radiography and computed tomography scout films for identifying renal calculi. BJU Int 2009, 104(5):670-673.

49. Kishore TA: Estimation of size of distal ureteral stone: non-contrast CT scan versus actual size. Urology 2008, 72(4):761-764.

50. Boll DT, Patil NA, Paulson EK, Merkle EM, Simmons WN, Pierre SA, Preminger GM: Renal stone assessment with dual-energy multidetector CT and advanced postprocessing techniques: improved characterization of renal stone composition-pilot study. Radiology 2009, 250(3):813-820.

51. Park J: Dual-energy computed tomography applications in uroradiology. Curr Urol Rep 2011, 16(3):178-183

52. Yang J, Yang S, Hsu H, Huang W: Transvaginal sonography in the morphological and functional assessment of segmental dilation of the distal ureter. Ultrasound Obstet Gynecol 2006, 27:449-451.

53. Mitterberger M, Pinggera G, Maier $E$, et al: Value of 3-dimensional transrectal/transvaginal sonography in diagnosis of distal ureteral calculi. J Ultrasound Med 2007, 26:19-27.

54. Shah A: Novel ultrasound method to reposition kidney stones. Urol Res 2010, 38(6):491-495. Epub 2010 Oct 22.

55. Sung MK: Current status of low dose multi-detector $C T$ in the urinary tract. World J Radiol 2011, 3(11):256-265.

56. John BS, Patel $U$, Anson $\mathrm{K}$ : What radiation exposure can a patient expect during a single stone episode? J Endourol 2008, 22(3):419-422.

57. Ferrandino MN, Bagrodia A, Pierre SA, Scales CD Jr, Rampersaud E, Pearle MS: Dual energy computed tomography with advanced postimage acquisition data processing: improved determination of urinary stone composition. J Endourol 2010, 24(3):347-54.

58. Preminger GM: Radiation exposure in the acute and short-term management of urolithiasis at two academic centers. J Urol 2009, 181(2):668-672.

59. Poletti P, Platon A, Rutschmann O: Low-dose versus standard-dose CT protocol in patients with clinically suspected renal colic. AJR Am J Roentgenol 2007, 188:927-933.

60. McCollough C, Bruesewitz M, Kofler J: CT dose reduction and dose managementtools: overview of available options. Radiographics 2006, 26:503-512. 
61. Memarsadeghi M, Heinz-Peer $G$, Helbich TH, et al: Unenhanced multidetector row $C T$ in patients suspected of having urinary stone disease: effect of section width on diagnosis. Radiology 2005, 235:530-536.

62. Ciaschini MW, Remer EM, Baker ME, Lieber M, Herts BR: Urinary calculi: radiation dose reduction of $50 \%$ and $75 \%$ at CT-effect on sensitivity. Radiology 2009, 251(1):105-111.

63. Jellison FC, Smith JC, Heldt JP, Spengler NM, Nicolay LI, Ruckle HC, Koning $J$, Millard WW, Jin DH, Baldwin DD: Effect of low dose radiation computerized tomography protocols on distal ureteral calculus detection. J Urol 2009, 182:2762-2767.

64. Jin DH: Effect of reduced radiation $\mathrm{CT}$ protocols on the detection of renal calculi. Radiology 2010, 255(1):100-107.

65. Karmazyn B, Frush DP, Applegate KE, Maxfield C, Cohen MD, Jones RP: CT with a computer-simulated dose reduction technique for detection of pediatric nephroureterolithiasis: comparison of standard and reduced radiation doses. Am J Roentgenol 2009, 192(1):143-149.

66. Krambeck AE: Diabetes mellitus and hypertension associated with shock wave lithotripsy of renal and proximal ureteral stones at 19 years of follow up. J Urol 2006, 175(5):1742-1747.

67. Chew BH: Twenty-year prevalence of diabetes mellitus and hypertension in patients receiving shock-wave lithotripsy for urolithiasis. BJU Int 2011, 22(4):268-274

68. Lee JY: Evaluation of the optimal frequency of and pretreatment with shock waves in patients with renal stones. Korean J Urol 2011, 52(11):776-781.

69. Mazzucchi E: Comparison between two shock wave regimens using frequencies of 60 and 90 impulses per minute for urinary stones. Clinics (Sao Paulo) 2010, 65(10):961-965.

70. Chacko J: Does a slower treatment rate impact the efficacy of extracorporeal shock wave lithotripsy for solitary kidney or ureteral stones? J Urol 2006, 175(4):1370-1373.

71. Evan AP: Renal injury during shock wave lithotripsy is significantly reduced by slowing the rate of shock wave delivery. BJU Int 2007 100(3):624-628.

72. Tham LM: Enhanced kidney stone fragmentation by short delay tandem conventional and modified lithotriptor shock waves: a numerical analysis. J Urol 2007, 178(1):314-319.

73. Maloney ME: Progressive increase of lithotripter output produces better in vivo stone comminution. J Endourol 2006, 20(9):603-606.

74. Willis LR: Prevention of lithotripsy- induced renal injury by pretreating kidneys with low -energy shock waves. J Am Soc Nephrol 2006, 17(3):663-673.

75. Kanao K: Preoperative nomograms for predicting stone-free rate after extracorporeal shock wave lithotripsy. $j$ Urol 2006, 176:1453-1457.

76. Nakajima Y, Kanao K: Current topics in the management of urinary tract stones; preoperative nomograms for predicting stone-free rate after ESWL Proceedings of 29th annual jackson hole urologic conference.

77. Vakalopoulos I: Development of a mathematical model to predict extracorporeal shockwave lithotripsy outcome. J Endouro/ 2009, 23(6):891-897

78. Wiesenthal JD: A clinical nomogram to predict the successful shock wave lithotripsy of renal and ureteral calculi. J Urol 2011, 186(2):556-562.

79. Shen P: Use of ureteral stent in extracorporeal shock wave lithotripsy for upper urinary calculi: a systematic review and meta-analysis. J Urol 2011, 186(4):1328-1335.

80. El-Assmy A: Impact of the degree of hydronephrosis on the efficacy of in situ extracorporeal shock-wave lithotripsy for proximal ureteral calculi. Scand J Urol Nephrol 2007, 41:208-213.

81. Alon Z: New concepts in shock wave lithotripsy. Urol Clin N Am 2007 34:375-382.

82. García Marchiñena P: CT SCAN as a predictor of composition and fragility of urinary lithiasis treated with extracorporeal shock wave lithotripsy in vitro. Arch Esp Urol 2009, 62(3):215-222. discussion 222

83. Leycamm L: Observations on intrarenal geometry of the lower caliceal system in relation to clearance of stone fragments after extracorporeal shockwave lithotripsy. J endourol 2007, 21(4):386-392.

84. Madaan S: Limitations of extracorporeal shock wave lithotripsy. Curr Opin urol 2007, 17(2):109-113.

85. Sapozhnikov OA: A mechanistic analysis of stone fracture in lithotripsy. J Acoust Soc Am 2007, 121(2):1190-1202.

86. da Silva SF R: Composition of kidney stone fragments obtained after extracorporeal shock wave lithotripsy. Clin Chem Lab Med 2010, 48(3):403-404
87. Schuler TD: Medical expulsive therapy as an adjunct to improve shockwave lithotripsy outcomes: a systematic review and meta-analysis. J Endourol 2009, 23(3):387-393.

88. Micali S: Can Phyllanthus niruri affect the efficacy of extracorporeal shock wave lithotripsy for renal stones? A randomized, prospective, long term study. J Urol 2006, 176(3):1020-1022.

89. Micali S, Micali S: Efficacy of expulsive therapy using nifedipine or tamsulosin, both associated with ketoprofene, after shock wavw lithotripsy of ureteral stones. Urol Res 2007, 178(43):89-93.

90. Zheng $\mathrm{S}$, et al: Tamsulosin as adjunctive treatmen after shockwave lithitripsy in patients with upper urinary tract stones: a systemic review and meta-analysis. Scand J Urol Nephrol 2010, 44:425-432.

91. Falahatkar S: Is there a role for tamsulosin after shock wave lithotripsy in the treatment of renal and ureteral calculi? J Endourology 2011,

25(3): 495-498.

92. Sighinolfi MC: Efficacy of tamsulsin treatment after extracorporeal shock wave lithotripsy of stone located in the kidney: a prospective and randomized study on 129 patient: $28^{\circ}$ World Congress of ndourology and SWL; 2010 Abstract.

93. Kijvikai K, Haleblian GE, Preminger GM, de la Rosette J: Shock wave lithotripsy or ureteroscopy for the management of proximal ureteral calculi: an old discussion revisited. J Urol 2007.

94. Aboumarzouk OM: Extracorporeal shock wave lithotripsy (ESWL) versus ureteroscopic management for ureteric calculi. Cochrane Database Syst Rev 2011, 12:CD006029.

95. Semins MJ: Management of stone disease in pregnancy. Curr Opin Urol 2010, 20(2):174-177.

96. Athanasios N: Optimizing shock wave lithotripsy in the $21^{\circ}$ century. Eur Urol 2007, 52:344-354

97. Geavlete P: flexible ureteroscopy: reshaping the upper urinary tract endourology. Arch Esp Urol 2011, 64(1):3-13.

98. Traxer O, Dubosg F, Jamali K, Gattegno B, Thibault P: New-generation flexible ureterorenoscopes are more durable than previous ones. Urology 2006, 68(2):276-279.

99. Monga M, Best S, Venkatesh R, Ames C, Lee C, Kuskowski M, Shwartz S, Vanlangendock R, Skepazy J, Landman J: Durability of flexible ureteroscopes: a randomized, prospective study. J Urol 2006, 176(1):137-141.

100. Canes D, Desai MM: New technology in the treatment of nephrolithiasis. Curr Opin Urol, 18:235-240

101. Paffen ML: A comparison of the physical properties of four new generation flexible ureteroscopes: (de)flection, flow properties, torsion stiffness, and optical characteristics. Endourol 2008, 22(10):2227-2234.

102. Haberman K: A dual-channel flexible ureteroscope: evaluation of deflection, flow, illumination, and optics. J Endourol 2011, 25(9):1411-1414. Epub 2011 Jul 28

103. Wendt-Nordahl G: Do new generation flexible ureterorenoscopes offer a higher treatment success than their predecessors? Urol Res 2011, 39(3):185-188

104. Multescu R: Conventional fiberoptic flexible ureteroscope versus fourth generation digital flexible ureteroscope: a critical comparison. J Endourol 2010, 24(1):17-21.

105. Jiang $H$, Wu Z, Ding $Q$, Zhang Y: Ureteroscopic treatment of ureteral calculi with holmium: YAG laser lithotripsy. J Endourol 2007, 21(2):151-154.

106. Binbay M: Evaluation of pneumatic versus holmium: YAG laser lithotripsy for impacted ureteral stones. Int Urol Nephrol 2011, 43(4):989-995

107. Hyams ES: Flexible ureterorenoscopy and holmium laser lithotripsy for the management of renal stone burdens that measure 2 to $3 \mathrm{~cm}$ : a multi-institutional experience. J Endourol 2010, 24(10):1583-1588.

108. Blew BD, Dagnone AJ, Fazio LM, et al: Practical comparison of four nitinol stone baskets. J Endourol 2007, 21:655-658.

109. Korman $\mathrm{E}$ : Comparison of small diameter stone baskets in an in vitro caliceal and ureteral model. J Endourol 2011, 25(1):123-127.

110. Morris DS, Wei JT, Taub DA, et al: Temporal trends in the use of percutaneous nephrolithotomy. J Urol 2006, 175:1731-1736.

111. Galvin DJ, Pearle MS: The contemporary management of renal and ureteric calculi. BJU Int 2006, 98(6):1283-1288.

112. Méndez Probst CE: Preoperative indications for percutaneous nephrolithotripsy in 2009. J Endourol 2009, 23(10):1557-1561. 
113. Valdivia JG: Supine versus prone position during percutaneous nephrolithotomy: a report from the clinical research office of the endourological society percutaneous nephrolithotomy global study. J Endourol 2011, 25(10):1619-1625.

114. Duty B: The debate over percutaneous nephrolithotomy positioning: a comprehensive review. J Urol 2011, 186(1):20-25. Epub 2011 May 14.

115. Ibarluzea G: Supine Valdivia and modified lithotomy position for simultaneous anterograde and retrograde endourological access. BJU Int 2007, 100(1):233-236.

116. Cracco CM: ECIRS (endoscopic combined intrarenal surgery) in the galdakao-modified supine valdivia position: a new life for percutaneous surgery? World J Urol 2011, 29(6):821-827.

117. Kawahara TB: Ureteroscopy assisted retrograde nephrostomy: a new technique for percutaneous nephrolithotomy (PCNL). JU Int 2011 , 111(67):123-127.

118. Wong C, Leveillee RJ: Single upper-pole percutaneous access for treatment of $>$ or $1 / 45-\mathrm{cm}$ complex branched staghorn calculi: is shockwave lithotripsy necessary? J Endourol 2002, 16:477-481.

119. Undre S, Olsen S, Mustafa N, Patel A: Pass the ball! Simultaneous flexible nephroscopy and retrograde intrarenal surgery for large residual upper-pole stag horn stone. J Endourol 2004, 18:844-847.

120. Ganpule AP: Multiperc versus single perc with flexible instrumentation for staghorn calculi. J Endourol 2009, 23(10):1675-1678.

121. Akman T: Comparison of outcomes after percutaneous nephrolithotomy of staghorn calculi in those with single and multiple accesses. J Endourol 2010, 24(6):955-960.

122. Li HL: Percutaneous nephrolithotomy through the upper pole calix access for complicated renal calculi: report of 581 cases. Nan Fang Yi Ke Da Xue Xue Bao 2011, 31(12):2079-2081.

123. Gokten OE: Efficacy of levobupivacaine infiltration to nephrosthomy tract in combination with intravenous paracetamol on postoperative analgesia in percutaneous nephrolithotomy patients. J Endourol 2011, 25(1):35-39.

124. Chen Y: Minimally invasive percutaneous nephrolithotomy under peritubal local infiltration anesthesia. World J Urol 2011, 29(6):773-777. Epub 2011 Jul 22.

125. Bove $P$ : Is telesurgery a new reality? Our experience with laparoscopic and percutaneous procedure. J Endourol 2003, 17(3):137-142.

126. Yates D: From Leonardo to da Vinci: the history of robot-assisted surgery in urology. RBJU Int 2011, 108(11):1708-1713.

127. Desai MM, Aron M, Gill IS, Pascal-Haber G, Ukimura O, Kaouk JH, Stahler G, Barbagli F, Carlson C, Moll F: Flexible robotic retrograde renoscopy: description of novel robotic device and preliminary laboratory experience. Urology 2008, 72(1):42-46.

128. Desai MM: Robotic flexible ureteroscopy for renal calculi: initial clinical experience. J Urol 2011, 186(2):563-568.

129. Mozer P, Troccaz J, Stoianovici D: Urologic robots and future directions. Curr Opin Urol 2009, 19(1):114-119.

130. Mariani A: Ureteroscopic monotherapy of large $(>2 \mathrm{~cm})$ renal calculi. J Endourol 2008, 22(Suppl 1):202.

doi:10.1186/1471-2490-13-10

Cite this article as: Rosa et al:: Recent finding and new technologies in nephrolithiasis: a review of the recent literature. BMC Urology 2013 13:10.

\section{Submit your next manuscript to BioMed Central and take full advantage of:}

- Convenient online submission

- Thorough peer review

- No space constraints or color figure charges

- Immediate publication on acceptance

- Inclusion in PubMed, CAS, Scopus and Google Scholar

- Research which is freely available for redistribution 